\title{
Structure of the Near-Injector Region of Nonevaporating Pressure-Atomized Sprays
}

\author{
G. A. Ruff, ${ }^{*}$ L. P. Bernal, $\dagger$ and G. M. Faeth $\ddagger$ \\ University of Michigan, Ann Arbor, Michigan 48109
}

\begin{abstract}
The dense-spray region of pressure-atomized nonevaporating sprays was studied, emphasizing the properties of the multiphase mixing layer that surrounds the liquid core during atomization breakup. The dispersed-phase properties of a large-scale $(9.5-\mathrm{mm}$ injector diameter) water jet injected vertically downward in still air were measured using single- and double-pulse holography for both fully developed and slug flow jet exit conditions. The inner portion of the mixing layer contained large irregularly shaped liquid elements and drops, and the proportion of spherical drops increased and drop sizes decreased with increasing radial distance. For present test conditions, the liquid core and the large liquid elements cause mean liquid volume fractions to be high near the axis; however, the gas-containing region was relatively dilute at each instant. Additionally, the velocities of large drops were generally much larger than small drops and predictions based on the locally homogeneous flow approximation, providing direct evidence of significant separated-flow effects in the flow. Finally, the degree of flow development at the jet exit had a substantial effect on the structure of the mixing layer, with increased turbulence levels increasing the number and size of large irregular liquid elements through distortion of the surface of the liquid core-enhancing rates of removal of liquid from the core.
\end{abstract}

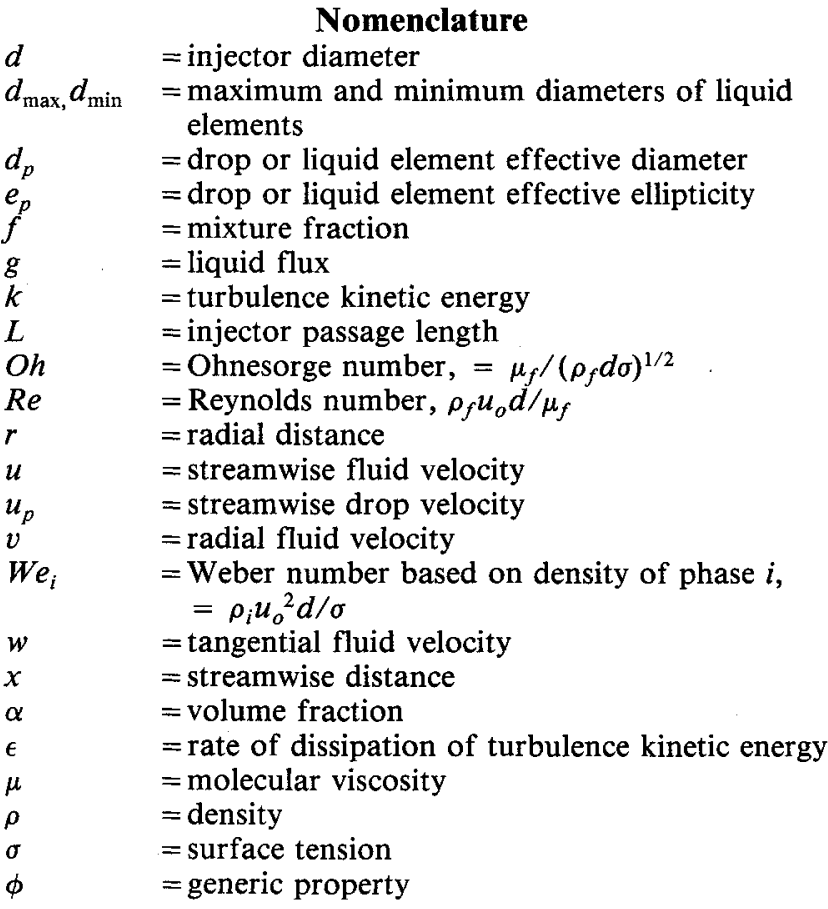

Subscripts

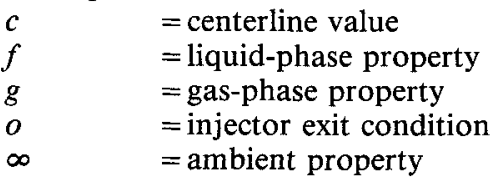

Presented as Paper 89-0050 at the AIAA 27th Aerospace Sciences Meeting, Reno, NV, Jan. 9-12, 1989; received May 18, 1989; revision received Nov. 30, 1989. Copyright (C) 1988 by the American Institute of Aeronautics and Astronautics, Inc. All rights reserved.

*Graduate Assistant, Department of Aerospace Engineering; currently Assistant Professor of Mechanical Engineering, Drexel University, Philadelphia, PA.

$\dagger$ Assistant Professor, Department of Aerospace Engineering.

$\ddagger$ Professor, Department of Aerospace Engineering. Fellow AIAA.

\begin{abstract}
Superscripts
()$^{-},\left(^{\prime}\right)^{\prime}=$ time-averaged mean and root-mean-squared fluctuating quantities

$(\tilde{)},(\tilde{C})=$ Favre-averaged mean and root-mean-squared fluctuating quantities
\end{abstract}

\section{Introduction}

$\mathbf{T}$ HIS investigation considered the dense-spray region near the injector of nonevaporating pressure-atomized sprays, e.g., round water jets injected into still air. The flow is of interest as it is the multiphase counterpart of the single-phase round turbulent jet; however, it also has numerous practical applications for propulsion and power systems, e.g., pressure atomization is used for fuel or propellant injectors of afterburners, liquid rocket engines, and fuel-injected internal combustion engines. Earlier measurements of liquid volume fractions and entrainment rates in the dense-spray region, reported by Ruff et al., ${ }^{1}$ were extended to provide the properties of the dispersed phase using single- and double-pulse holography. The new measurements were used to continue evaluation of analysis of the flow based on the locally homogeneous flow (LHF) approximation of multiphase flow theory, i.e., the assumption that interphase transport rates are infinitely fast so that both phases have the same instantaneous velocity and are in thermodynamic equilibrium at each point in the flow. Present considerations were limited to the near-injector region, within the atomization breakup regime as defined by Ranz, ${ }^{2}$ since this breakup regime is of greatest importance for practical applications. Similar to Ruff et al.,' both slug flow and fully developed turbulent pipe flow jet exit conditions were considered in order to study effects of flow development at the jet exit on the structure of dense pressure-atomized sprays.

Earlier studies of the dense-spray region of pressureatomized sprays have been reviewed recently ${ }^{3}$; therefore, only their main features will be considered here. Current understanding is relatively limited due to difficulties of measuring, or even optically penetrating, a multiphase flow when liquid volume fractions vary over a wide range. Nevertheless, it is generally agreed that the near-injector region involves a liquid core, somewhat like the potential core of single-phase jets, surrounded by a multiphase mixing layer that begins to develop right at the injector exit for flows in the atomization 
breakup regime. The dense-spray region is normally considered to include both the liquid core and the multiphase mixing layer-extending from the injector exit to the point where the liquid core disappears. This region can extend quite far from the injector, e.g., the liquid core is still present $200-400$ injector diameters from the jet exit for injection into gases at atmospheric pressure, depending on velocity profiles and turbulence levels at the injector exit. ${ }^{4-6}$

The dense-spray region involves widely varying phase topography-the liquid core, irregular liquid elements, ligaments and drops-and a variety of physical phenomenabreakup, collisions, interphase transport from irregular liquid elements, etc. In an attempt to circumvent these complexities, a number of workers have studied the use of the LHF approximation to analyze dense sprays. LHF analysis circumvents the need for separated-flow parameters that are poorly understood for dense sprays; however, the effectiveness of the approach is controversial. Bracco ${ }^{7}$ and $\mathrm{Wu}$ et al.$^{8,9}$ report measurements of spray angles and drop velocities in nonevaporating pressure-atomized sprays at high pressures, concluding that LHF analysis was effective for their test conditions. On the other hand, earlier work in this laboratory indicated that the LHF approach generally overestimates the rate of development of multiphase jets and, although it provides useful qualitative information, it is not quantitatively accurate for most practical sprays. ${ }^{10-17}$ Even results of LHF analysis for sprays in high-pressure gases were not satisfactory since drop inertia is significant in the rapidly decelerating flowfield of typical sprays due to relatively small injector diameters and fast rates of entrainment. ${ }^{10,11}$ Experimental evidence on both sides of the controversy comes from dilute portions of sprays and particle-laden flows, however, and the relevance of this information to dense-spray processes is questionable.

The first phase of the present investigation sought to help resolve controversies concerning the structure and extent of the dense-spray region and the effectiveness of analysis of dense sprays based on the LHF approximation. ${ }^{1}$ Experiments were undertaken with large-scale $(9.5$ - and 19.1-mm-diam injectors having both fully developed and slug flows at the injector exit) nonevaporating water jets in still air at atmospheric pressure. Operation in both the wind-induced and atomization breakup regimes was considered. The following measurements were made: flow visualization using flash photography, timeaveraged liquid volume fractions using gamma-ray absorption, and air-entrainment rates and mean and fluctuating liquid velocities at the jet exit using laser velocimetry (LV). Measurements were compared with predictions based on the LHF approximation along the lines of Mao et al., ${ }^{10,11}$ after calibration of estimates of turbulent mixing rates based on measurements in single-phase variable-density jets. ${ }^{3}$

The findings of Ruff et al. ${ }^{1}$ have helped to clarify some issues of dense sprays. Measurements of mean liquid volume fractions showed that the initial rate of development of the flow, and the length of the liquid core, are strongly dependent on both the degree of flow development at the jet exit and the breakup regime, with fully developed flow and atomization breakup yielding the fastest rates of flow development. This sensitivity of the flow to jet exit conditions highlights problems of using small injectors to study dense sprays because it is difficult to provide well-defined jet exit conditions when small injector passages are used. Predictions of mean liquid volume fractions based on the LHF approximation were reasonably good within the near-injector region for atomization breakup, including correct predictions of the sensitivity of the flow to the degree of flow development at the jet exit. However, predictions began to significantly overestimate the rate of development of the flow as the spray became dilute, i.e., for mean liquid volume fractions along the axis $<0.2$. Furthermore, the theory provided no warning of the vastly reduced mixing rates that were observed when the flow was in the windinduced breakup regime (where formation of the multiphase mixing layer begins downstream of the jet exit and drop sizes are generally larger than for atomization breakup). Finally, the entrainment rates of the sprays were generally overestimated for all conditions, although predictions tended to improve as Reynolds numbers were increased in the atomization breakup regime. This behavior was attributed to the poorer performance of LHF analysis in dilute sprays since entrainment is dominated by the mixing properties of the dilute-spray region near the outer edge of the multiphase mixing layer. The findings generally suggested that the LHF approximation represents behavior approaching the limit of infinitely large jet Reynolds numbers, ${ }^{18,19}$ where drops become infinitely small, concluding that practical sprays invariably involve effects of separated flow-particularly in the dilute-spray regions of the flow.

The present investigation extends the study of Ruff et al., ${ }^{1}$ emphasizing separated-flow phenomena within the multiphase mixing layer near the injector exit. Measurements were carried out using the same apparatus but with test conditions limited to the atomization breakup regime. Visualization of the flow was emphasized in order to provide direct information on the topography of the dispersed phase within the multiphase mixing layer. This was accomplished using single- and doublepulse holography to yield drop size and velocity distributions, the character of irregular liquid elements found near the liquid core, and distributions of liquid volume fractions and fluxes. Similar to Ruff et al., ${ }^{1}$ measurements were compared with predictions based on the LHF approximation to help provide a measure of separated-flow effects.

The paper begins with descriptions of experimental methods and the main features of the LHF analysis. The paper concludes with descriptions of experimental and theoretical findings, considering the appearance of the flow, liquid element sizes and velocities, and liquid volume fractions and fluxes, within the multiphase mixing layer. The present discussion is brief; additional details can be found in Ruff and Faeth ${ }^{20}$ and Ruff. ${ }^{21}$

\section{Experimental Methods}

\section{Apparatus}

Based on measurements of drop-size distributions in dilute sprays, an effect of injector diameter on dense-spray properties can be anticipated but it is not strong. ${ }^{22}$ Thus, large-scale (9.5-mm injector diameter) jets were used in order to provide adequate spatial resolution for observations. Water was used as the test liquid, injected vertically downward in still air. The water was collected in a baffled tub, to prevent splashing up into the area where measurements were made, and discharged to a drain. City water was supplied to the injector by a centrifugal pump. The water flow rate was adjusted using a bypass system and measured with a turbine flow meter that was calibrated by collecting water for timed intervals.

The slug and fully developed flow injectors were the same as the ones used by Ruff et al. ${ }^{1}$ Both injectors had exit diameters of $9.5 \mathrm{~mm}$. The slug flow injector involved a honeycomb flow straightener and two screens followed by a 13.6:1 area contraction designed to provide uniform flow at the exit. The fully developed flow injector used the same flow straightener and contraction ratio but this was followed by a constant-area passage having a length-to-diameter ratio of 41 . Mean streamwise velocities and streamwise and cross-stream velocity fluctuations were measured across the exit of the injectors using laser velocimetry (see Ref. 21 for details of the measurements and plots of velocity profiles). The results show that the slug flow injector provided relatively uniform velocities with a streamwise turbulence intensity of roughly $1 \%$ at the jet exit, whereas fully developed flow injectors yielded properties at the jet exit that were in reasonably good agreement with existing measurements for fully developed turbulent pipe flow..$^{23,24}$ Instrumentation was mounted rigidly; therefore, the flow structure was measured by traversing the injector horizontally (up to $1 \mathrm{~m}$ with a positioning accuracy of $5 \mu \mathrm{m}$ ) and vertically (up to $2 \mathrm{~m}$ with a positioning accuracy of $0.5 \mathrm{~mm}$ ). 


\section{Instrumentation}

Present measurements were limited to dispersed-phase properties in the multiphase mixing layer using holography. An off-axis holographic arrangement was used based on the Spectron Development Laboratories Model HTRC-5000 system, with an angle of $28 \mathrm{deg}$ between the object and reference beams. The system was modified, however, to improve performance in dense sprays. In particular, dense sprays require large light intensities so that the flow can be penetrated and high magnification since small drops are of interest. This was accomplished by reducing the diameter of the object beam through the spray and then subsequently expanding it (7-8:1) back to the same size as the reference beam $(85-\mathrm{mm}$ diam) when the two signals were optically mixed to form a hologram. The reference beam was directed past the spray within a 300 -mm-diam tube to reduce optical noise from small drops in the environment caused by the spray collection system. The holograms were produced using a ruby laser that deposited 50 $\mathrm{mJ}$ in roughly $20 \mathrm{~ns}$. The short laser pulse time stopped the motion adequately so that drops as small as $2 \mu \mathrm{m}$ in diameter could be observed and drops as small as $5 \mu \mathrm{m}$ in diameter could be measured. The laser could be double pulsed with pulse separation times as short as $2 \mu \mathrm{s}$. This yielded holograms that could be reconstructed to show the object field at two instants of time, providing a means of measuring liquid element velocities. The intensity of the two pulses differed and was monitored with a photodiode. This provided a means of determining the direction of drop motion since the pulse having greater intensity yielded a higher contrast image. The holograms were obtained in a darkened room using AGFA 8E75HD-NAH unbacked holographic film plates with a 100 $\times 125$-mm film format.

The holograms were reconstructed using a $15-\mathrm{mW} \mathrm{cw} \mathrm{HeNe}$ laser that was collimated at a $60-\mathrm{mm}$ diam and passed through the developed hologram to provide a real image of the spray in front of the hologram. The properties of the reconstructed spray were observed with an MTI Model 65 video camera with optics that provided fields of view of roughly $1 \times 1.2 \mathrm{~mm}$ and $2.5 \times 3 \mathrm{~mm}$. The larger field of view was more convenient for analyzing ligaments and other large liquid elements. Computer-controlled $x-y$ traversing of the hologram (with a $1-\mu \mathrm{m}$ resolution) and $z$ traversing of the video camera (with $5-\mu \mathrm{m}$ resolution) allowed the region crossed by the object beam to be studied. The video image was analyzed using a Gould FD 5000 Image Processing System. A 1-mm-diam pin located in the region being studied provided a size and position reference point in the reconstructed image of the spray.

Drops and other more-or-less ellipsoidal-shaped liquid elements were sized by finding the maximum and minimum diameter through the centroid of the image. Assuming that the liquid element was ellipsoidal, its diameter was taken to be the diameter of an ellipsoid having the same volume, i.e., $d_{p}^{3}=$ $d_{\min }^{2} d_{\max }$. The shape of the element was characterized by its ellipticity, defined as $e_{p}=d_{\max } / d_{\min }$. This approach was not appropriate for elongated liquid elements or ligaments where the centroid of the image often fell outside the boundaries of the image or where there were several necked-in points along the length of the element. In these cases, the cross-sectional area and perimeter of the image were measured and the maximum and minimum diameters of an ellipsoid having the same cross-sectional area and perimeter were computed. Given these parameters, the effective diameter and ellipticity were calculated in the same manner as for drops. Results at each position in the flow were summed to find the Sauter mean diameter (SMD), volume-averaged ellipticity, and liquid volume fraction.

Velocity measurements were based on the motion of the centroid of the image and were correlated as a function of diameter using a least-squares fit. This allowed plots of drop velocities at fixed diameters across the width of the mixing layer while making maximum use of data on the holograms at each position. Knowing the volume and velocity of liquid ele- ments in the flow, liquid fluxes could be computed in a straightforward manner as well.

Measurements were made at $x / d=12.5,25,50$, and 100 for $r / x$ extending from the surface of the liquid core to near the edge of the multiphase mixing layer. Measurements at each location were obtained from a $6 \times 6 \times 4-\mathrm{mm}$ volume, averaging results over no less than three holograms. The data were spatially averaged over the width of the measuring volumes, or $\pm 1 / 2$ the distance between adjacent radial positions, whichever was less. Measurements involved analysis of 50-150 objects for $r / x \leq 0.075$ and 150-500 objects for $r / x \geq 0.100$, the fewer objects nearer the liquid core reflecting the fact that liquid elements were generally much larger in this region.

Experimental uncertainties were generally dominated by sampling limitations rather than the resolution of liquid element properties from the reconstructed holograms. Estimates of experimental uncertainties $(95 \%$ confidence) are as follows: Sauter mean diameter $<10 \%$, volume-averaged ellipticity $<15 \%$, liquid element velocities $<20 \%$, liquid volume fractions $<15 \%$, and liquid fluxes less than $25 \%$.

\section{Test Conditions}

Mean flow conditions were the same for the slug and fully developed flows. The test conditions are summarized in Table 1. The injector Reynolds number was reasonably high, yielding a turbulent multiphase mixing layer. Ranz ${ }^{2}$ suggests $W e_{f}>8$ and $W e_{g}>13$ for atomization breakup, whereas Miesse $^{25}$ recommends $W e_{g}>40.3$ for atomization breakup; by either criterion the present flows were well into the atomization breakup regime that clearly corresponded to their appearance.

\section{Theoretical Methods}

Analysis of the flows was limited to the use of the LHF approximation along the lines of past work in this laboratory (see Faeth ${ }^{3}$ for a description of the general formulation). The main features of the analysis will be described in the following because the formulation was modified in order to reduce empiricism from the approach described by Ruff et al. ${ }^{1}$ and Ruff and Faeth ${ }^{20}$ for the same flows. In particular, it was found that the low levels of water evaporation in the test sprays had little effect on predictions ${ }^{1}$; therefore, this effect was ignored, passing to the limit of a nonevaporating spray.

In addition to the LHF approximation, the major assumptions of the analysis were as follows: steady (in the mean) axisymmetric flow with no swirl, boundary-layer approximations apply, negligible kinetic energy and viscous dissipation of the mean flow, buoyancy only affects the mean flow, equal exchange coefficients of all species and phases, and negligible mass transport between the phases. These assumptions are either conditions of the experiments or have been justified by past performance, with the exception of the LHF approximation that is to be evaluated here.

Under these assumptions, all scalar properties are only functions of mixture fraction (defined as the fraction of mass at a point that originated from the injector). Furthermore, the

Table 1 Summary of test conditions ${ }^{a}$

\begin{tabular}{ll}
\hline Injector diameter, mm & 9.5 \\
Flow rate, $\mathrm{kg} / \mathrm{s}$ & 3.99 \\
Injector pressure drop, kPa & \\
$\quad$ Fully developed flow & 2520 \\
$\quad$ Slug flow & 2270 \\
Average jet exit velocity, m/s & 56.3 \\
$R e$ & 534000 \\
$W e_{f}$ & 412000 \\
$W e_{g}$ & 500 \\
$O h$ & 0.00121 \\
\hline \hline
\end{tabular}

aPressure-atomized water jet injected vertically downward in still air at 98.8 $\mathrm{kPa}, 298 \pm 2 \mathrm{~K}$; in the atomization breakup regime for both slug flow and fully developed turbulent pipe flow $(L / d=41)$ jet exit conditions. 
instantaneous mixture fraction is either 0 or 1 since a particular point can be only in either gas $(f=0)$ or liquid $(f=1)$, i.e., the probability density function of mixture fraction consists of Dirac delta functions at $f=0$ and 1 . Taking scalar properties at the jet exit and in the environment to be $\phi_{o}$ and $\phi_{\infty}$, all mean scalar properties can then be found in terms of the Favreaveraged mean mixture fraction $\tilde{f}$ as follows:

$$
\begin{gathered}
\tilde{\phi}=\phi_{\infty}(1-\tilde{f})+\phi_{o} \tilde{f} \\
\bar{\phi}=\left(\phi_{\infty} \rho_{o}(1-\tilde{f})+\phi_{o} \rho_{\infty} \tilde{f}\right) /\left(\rho_{o}(1-\tilde{f})+\rho_{\infty} \tilde{f}\right)
\end{gathered}
$$

Similarly, higher moments of scalars- $\tilde{\phi}^{\prime \prime}, \bar{\phi}^{\prime}$, etc.-are also only functions of $\tilde{f}$ for present conditions.

Given Eqs. (1) and (2), the flowfield can be found using a simplified version of the conserved-scalar formalism of Lockwood and Naguib, ${ }^{26}$ but based on mass-weighted (Favre) averages, following Bilger. ${ }^{27}$ Governing equations are solved for conservation of mass, streamwise mean momentum, mean mixture fraction, turbulence kinetic energy, and the rate of dissipation of turbulence kinetic energy. Unlike the formulation of Ruff et al. ${ }^{1}$ and Ruff and Faeth ${ }^{20}$ (which is suitable for evaporating flow), however, there is no need to solve a governing equation for mean-squared mixture fraction fluctuations since scalar properties can be found in terms of $\tilde{f}$ from Eqs. (1) and (2). The specific formulation of the governing equations and all empirical constants can be found in Ref. 3. The approach was successfully calibrated for a variety of constant and variable density single-phase round jets ${ }^{28}$; the formulation and constants, however, are not very different from those used by Lockwood and Naguib. ${ }^{26}$

Initial conditions for the calculations were based on the LV measurements at the injector exit, similar to Ruff et al. ${ }^{1}$ For slug flow, flow properties were uniform at the exit, as noted earlier, except for a narrow layer that could not be resolved by the LV. In the constant property portion of the flow, $\bar{u}$ was known and $k$ was computed from measurements of $\bar{u}^{\prime 2}$ and $\bar{v}^{\prime 2}$ by assuming $\bar{w}^{\prime 2}=\bar{v}^{\prime 2}$, whereas $\epsilon$ was estimated to be $1.274 \times 10^{-4} \bar{u}_{o}^{3} / d$, similar to past work. ${ }^{3}$ Properties in the boundary layer along the wall were estimated for a range of $L / d$, bounding reasonable estimates of flow development lengths, assuming clean entry and no vena contracta, based on Schlichting. ${ }^{24}$ For fully developed flow, initial profiles of $\tilde{u}, k$, and $\epsilon$ for fully developed turbulent pipe flow were taken from Hinze $^{23}$ and Schlichting. ${ }^{24}$ In all cases, $\tilde{f}=1$ and Favre- and time-averaged quantities are identical in the single-phase flow at the injector exit.

The governing equations were solved using GENMIX. ${ }^{29}$ The large density variation of the flow caused problems of computational stability and numerical accuracy, requiring much finer grids than are usually needed for single-phase flows. Present computations used 720 cross-stream grid nodes with streamwise step sizes limited to $0.15 \%$ of the current flow width. Doubling the number of grid nodes in both the crossstream and streamwise directions changed predictions $<1 \%$.

\section{Flow Visualization \\ Results and Discussion}

Photographs of typical single-pulse hologram reconstructions, taken directly from the monitor of the video camera of the image processing system, are illustrated in Figs. 1-3. These results were obtained within the multiphase mixing layer at $x / d=12.5$ for fully developed flow; however, results at other axial stations and for slug flow are similar. Three radial positions are considered, corresponding to points near the outer edge (Fig. 1), middle (Fig. 2), and inner edge (Fig. 3) of the multiphase mixing layer. All three photographs were obtained with the same magnification, yielding a field of view of 1725 $\times 2250 \mu \mathrm{m}$. Except near the liquid surface, usually only a few drops or liquid elements (or portions of them) are in focus for any one screen image of the video monitor. Thus, the photo-

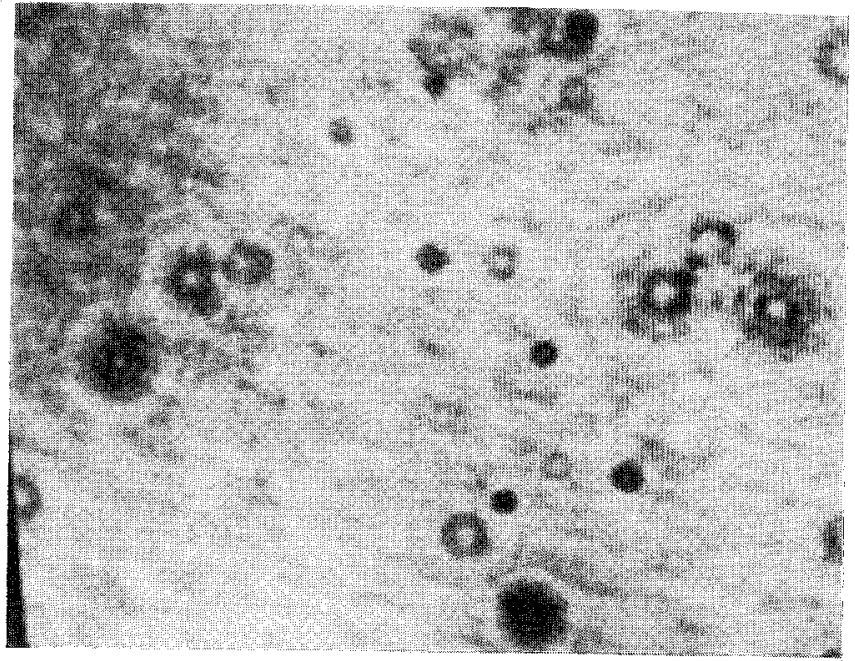

Fig. 1 Typical single-pulse hologram reconstruction near the outer edge of the multiphase mixing layer: fully developed flow, $x / d=$ 12.5, $r / x=0.150$.

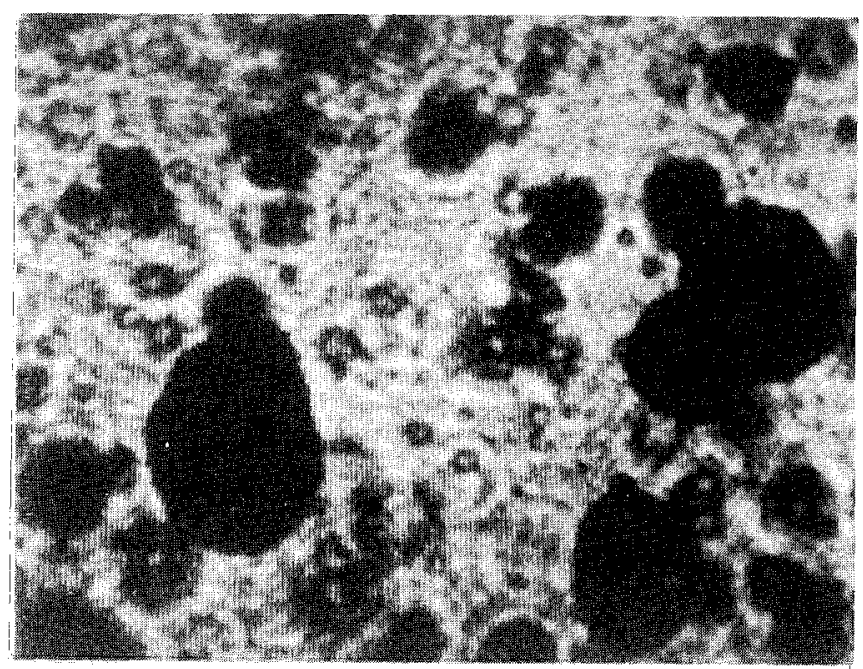

Fig. 2 Typical single-pulse hologram reconstruction near the middle of the multiphase mixing layer: fully developed flow, $x / d=12.5$, $r / x=0.100$.

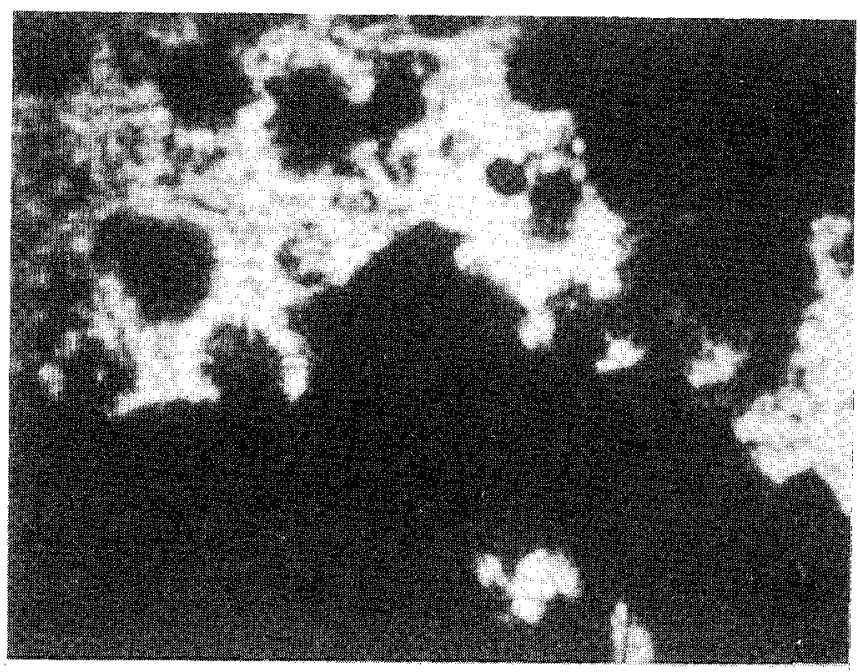

Fig. 3 Typical single-pulse hologram reconstruction near the inner edge of the multiphase mixing layer: fully developed flow, $x / d=$ 12.5, $r / x=0.050$. 
graphs have a mottled appearance due to out-of-focus drops. Furthermore, the images on the monitor are much larger and easier to interpret-particularly since the focal plane can be moved to sharpen the focus on any one object.

Conditions near the outer edge of the multiphase mixing layer, Fig. 1, correspond to a dilute spray: the liquid elements consist of relatively small spherical drops separated by rather large distances; therefore, the liquid volume fraction is quite small. Moving to the middle of the multiphase mixing layer, Fig. 2, the flow largely remains a dilute spray although drop diameters and drop number densities are larger than near the edge of the flow. In this region, however, some of the larger liquid elements are no longer spherical, although the ellipticities of individual liquid elements are rarely greater than 2 . Finally, as the edge of the liquid core is approached, Fig. 3, the liquid elements become quite large and are generally very irregular in shape, consisting of ligaments and long ellipsoids surrounded by only a few spherical drops. The lower right corner of Fig. 3 actually corresponds to a section of the surface of the liquid core. This surface is quite irregular and involves long liquid elements protruding into the mixing layer, suggesting initial growth of ligaments before they break away from the surface. A surprising feature of the region near the liquid core, however, is that the gas-containing region is relatively dilute at each instant. Even high-magnification reconstructions of this region did not reveal large numbers of small drops. Thus, the main difference between the dilute- and dense-spray regions of the multiphase mixing layer is that the dense-spray region contains large and irregular liquid elements, including protuberances from the liquid core. These

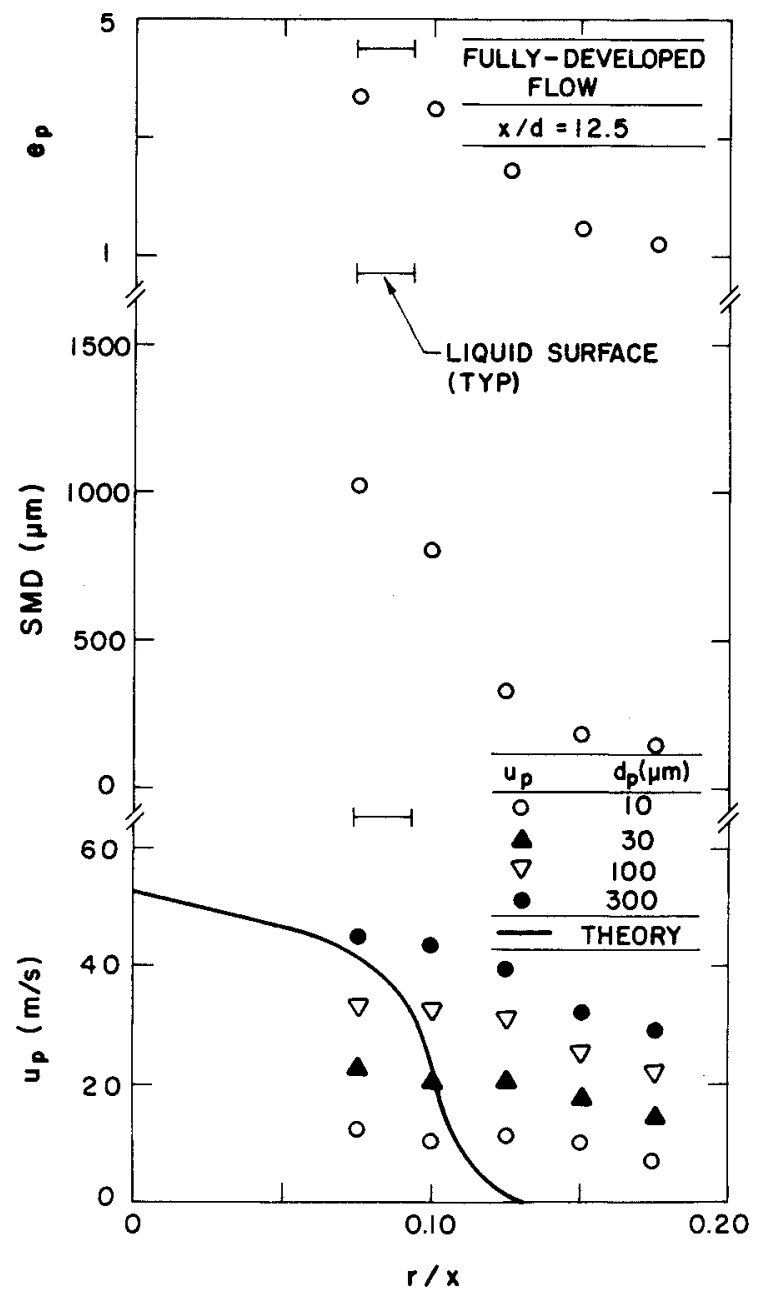

Fig. 4 Disperse-phase properties for fully developed flow at $x / d=12.5$. large liquid objects, as well as lateral fluctuations of the liquid core itself, cause time-averaged liquid volume fractions to be large even though the gas-containing region surrounding the liquid elements is relatively dilute at each instant.

\section{Liquid Element Sizes and Velocities \\ Fully Developed Flow}

Measurements of liquid element sizes and velocities within the multiphase mixing layer for fully developed flow at $x / d=$ $12.5,25,50$, and 100 are illustrated in Figs. 4-7. The volumeaveraged ellipticity, SMD, and drop velocities for $d_{p}=10,30$, 100 , and $300 \mu \mathrm{m}$, are plotted as a function of $r / x$, which is the radial similarity variable for turbulent single-phase jets and plumes. This radial similarity variable has only been chosen for convenience: the region considered is analogous to the mixing layer around the potential core of a single-phase jet due to the presence of the liquid core, and radial profiles for flow properties do not exhibit similarity in the $r / x$ coordinate system. The range of positions where the edge of the liquid core was observed is also marked on the plots: the measurements extend from the liquid core to near the outer edge of the multiphase flow region where $r / x$ is in the range $0.150-0.175$.

In Figs. 4-7, large values of volume-averaged ellipticity, which are associated with the presence of large and irregular liquid elements, are generally found in the region of the liquid core. This region extends across most of the multiphase mixing layer near the jet exit but becomes progressively confined to the region near the axis with increasing distance from the jet exit. Thus, the near-injector region of the spray can be divided

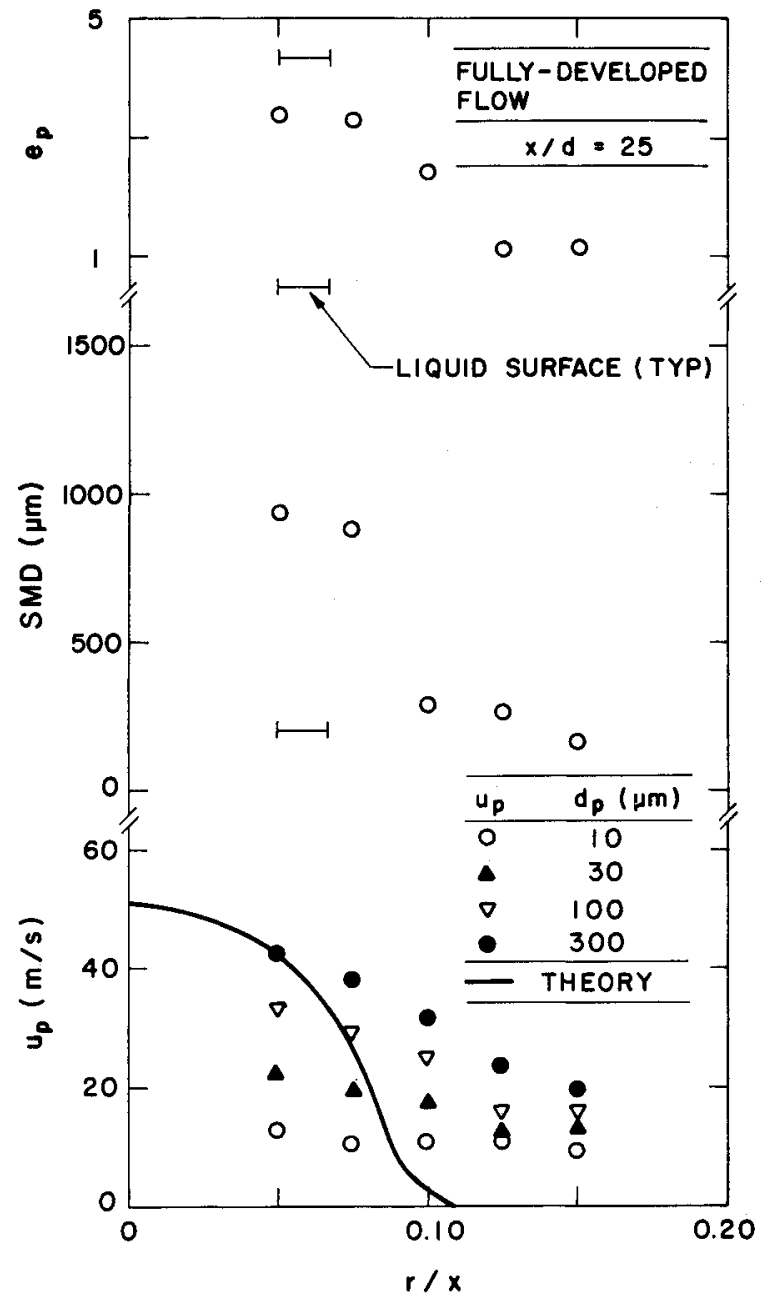

Fig. 5 Dispersed-phase properties for fully developed flow at $x / d=25$. 


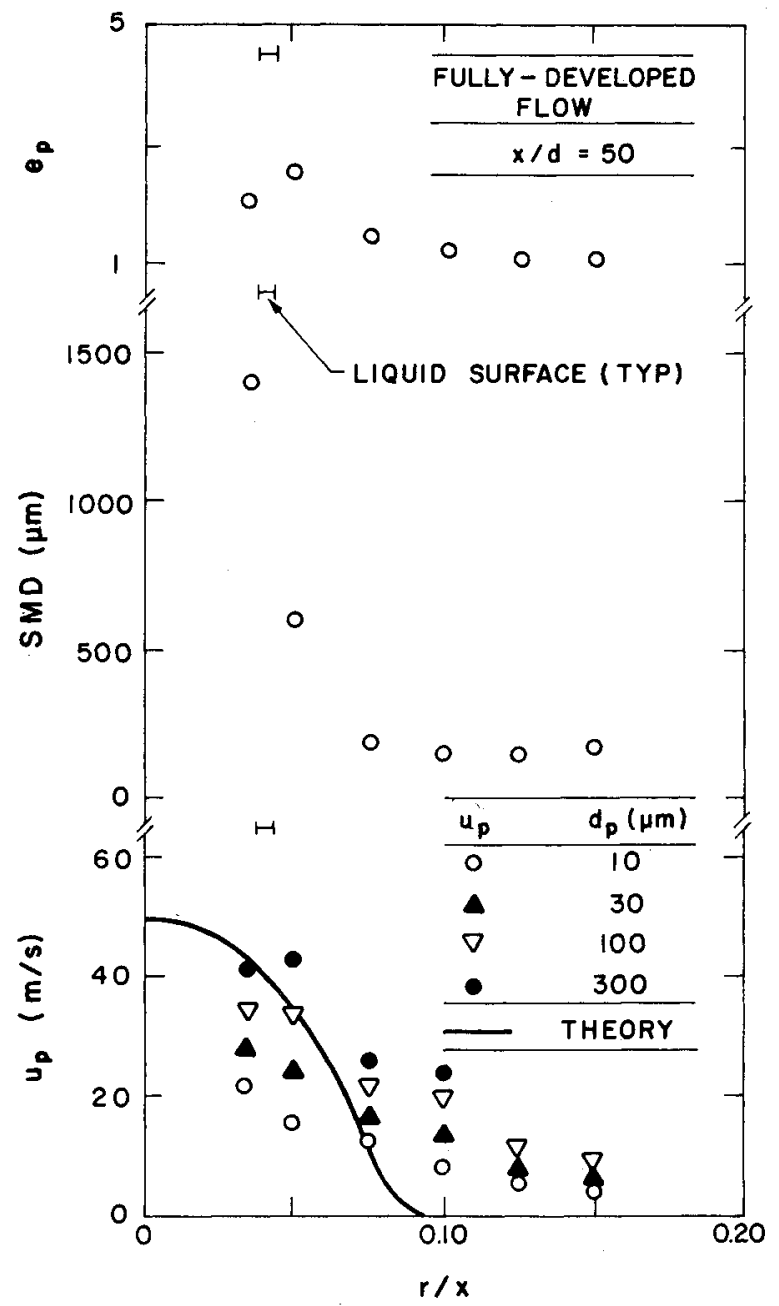

Fig. 6 Dispersed-phase properties for fully developed flow at $x / d=50$.

into three regions: a liquid core, a dense-spray region characterized by nonunity ellipticities, and an outer dilute-spray region where ellipticities are near unity. The outer region progressively grows with increasing distance from the injector, eventually reaching the axis somewhat downstream of the region where liquid stripping by the formation of ligaments and other large liquid elements has caused the liquid core to disappear.

Sauter mean diameters, illustrated in Figs. 4-7, progressively decrease with increasing radial distance, similar to the ellipticity. Values of the SMD near the liquid core are quite large, $600-1000 \mu \mathrm{m}$, due to the presence of large irregular liquid elements. Near the edge of the flow in the dilute-spray region, however, drop sizes are much smaller, generally in the range $100-200 \mu \mathrm{m}$, but dropping to even smaller values at the extreme outer edge of the multiphase mixing layer (see Fig. 7). Several phenomena are probably responsible for the progressive reduction of SMD with increasing radial distance: the tendency of large liquid elements to shatter as they encounter low-velocity gas near the edge of the flow, the fact that breakup of ligaments and other large liquid elements requires a finite time allowing them to traverse the inner portions of the mixing layer, and increased turbulent dispersion of small drops that enhances their migration toward the edge of the flow.

Distributions of drop velocities in Figs. 4-7 include both measurements for various drop diameters and predictions based on the LHF approximation. In the region near the liquid core, the largest drops have velocities that are comparable to liquid injection velocities, ca. $56 \mathrm{~m} / \mathrm{s}$. This follows since velocities within the liquid core remain relatively close to jet exit velocities, whereas the large drops have formed only recently

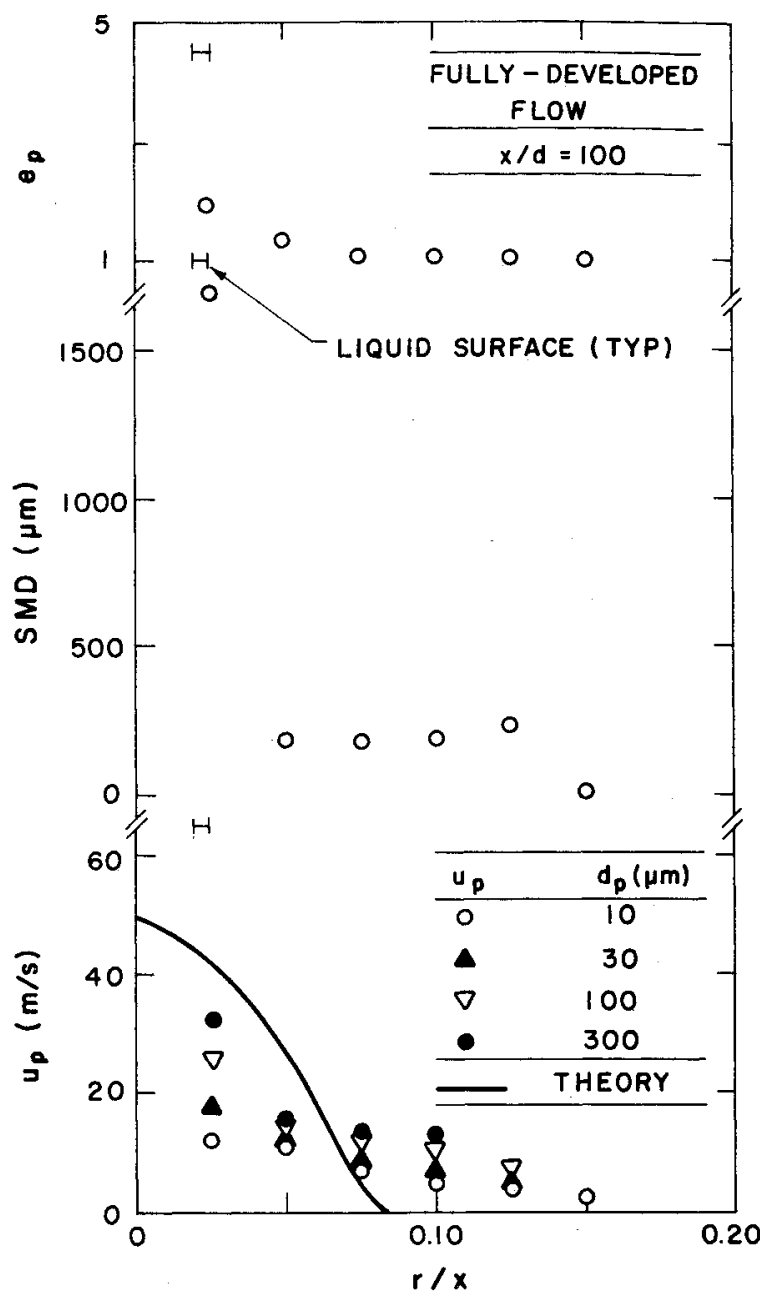

Fig. 7 Dispersed-phase properties for fully developed flow at $x / d=100$.

and have not had sufficient time for drag from the gas phase to slow their motion. The velocities of drops of all sizes, however, tend to decrease with increasing radial distance. This is expected since gas velocities are lowest near the edge of the flow and drops in this region have had more time to accommodate to gas velocities. A surprising feature of the results of Figs. 4-7, however, is that small drops (which should have velocities relatively close to local gas velocities) have relatively low velocities that are nearly constant across much of the mixing layer-particularly at $x / d=12.5$ and 25 (Figs. 4 and 5). This suggests that momentum exchange between the liquid and gas is not very efficient in the multiphase mixing layer, perhaps due to the presence of large liquid elements (having relatively low surface-to-volume ratios) in the dense-spray region near the liquid core. This results in relatively large relative velocities between the phases near the liquid core, which probably helps promote breakup.

The fact that the drop velocities plotted in Figs. 4-7 vary substantially with drop diameter at each point in the flow provides direct evidence that use of the LHF approximation is not appropriate for the present flow. In view of this, it is hardly surprising that the LHF predictions are not in good agreement with the measurements. The nature of the failure is typical of past evaluations of the LHF approximation for dilute sprays; namely, the rate of development of the flow is overestimated since the analysis does not properly account for the inertia of large drops that take significant time to exchange momentum with the gas phase. In view of the results of Figs. 4-7, it is also not surprising that the LHF approach tended to overestimate the rate of entrainment of the flow during the evaluation of Ref. 1. Under the LHF approximation, all of the momentum 


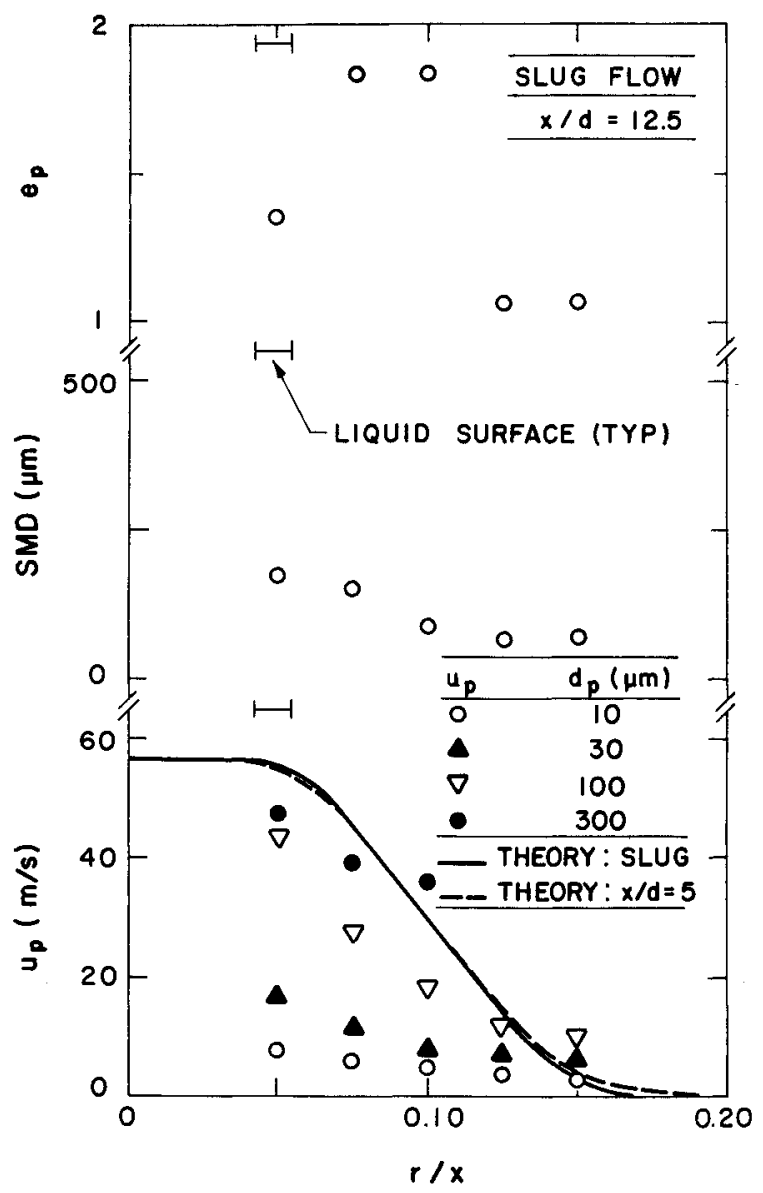

Fig. 8 Dispersed-phase properties for slug flow at $x / d=12.5$.

of the flow is available to the continuous phase to help promote mixing; however, since the drops have substantial relative velocities, their relative momentum is not available to the gas phase and the rate of mixing is reduced accordingly.

\section{Slug Flow}

Measurements of liquid element sizes and velocities within the multiphase mixing layer for slug flow are illustrated in Figs. 8-11. These figures are plotted in the same manner as Figs. 4-7 for fully developed flow except that the scales for ellipticity and SMD have been expanded to improve readability. Many features of dispersed-phase properties are the same for slug and fully developed flow: ellipticities are largest near the liquid surface, where ligaments and other large liquid elements are present, whereas the edge of the flow involves nearly round drops; drop sizes are largest near the liquid core, dropping to SMD in the range $100-200 \mu \mathrm{m}$ near the edge of the flow; and drop velocities vary substantially with drop size, whereas small drops, and probably the gas as well, have relatively low velocities near the liquid surface.

The main differences between the fully developed flow results of Figs. 4-7 and the slug flow results of Figs. 8-11 are that the ellipticities and SMD are smaller near the liquid core for slug flow. This suggests that breakup mechanisms at the liquid surface are modified by turbulence levels within the liquid core. Such behavior is quite reasonable based on flash photographs of the liquid surface (for wind-induced breakup where the surface can be readily seen) for fully developed and slug flow reported by Ruff et al. ${ }^{1}$ In particular, the surface was very wrinkled by the underlying turbulence in the liquid for fully developed flow, whereas the surface was smoother for slug flow. Clearly, the wrinkling present for fully developed flow would help promote the expulsion of large liquid elements into the gas phase, enhancing effects of liquid-jet instability that are present for both flows. Liquid-jet instability,

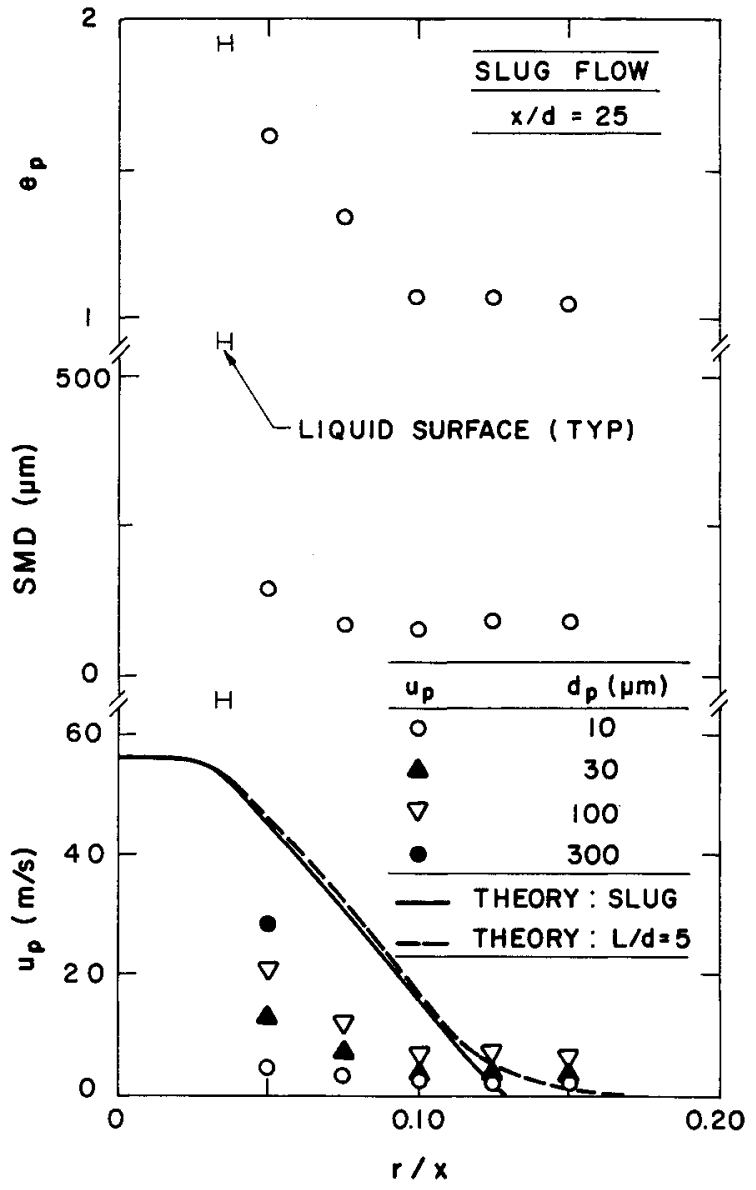

Fig. 9 Dispersed-phase properties for slug flow at $x / d=25$.

working alone, cannot cause breakup to penetrate as far into the liquid phase, yielding the smaller drops observed during the present study, as well as slower rates of mixing of the flow, observed by Ruff et al. ${ }^{1}$ Even though the drops are initially smaller for slug flow, however, secondary drop breakup processes in the gas phase are still important, as evidenced by reduced SMD near the edge of the multiphase mixing layer and at the position farthest from the injector.

Another difference between structure of the mixing layer for fully developed and slug flow is that the layer is significantly wider for fully developed flow. A mechanism for this behavior is lateral penetration of large liquid elements, which conserve outward radial velocity fluctuations present when they are expelled from the liquid due to their inertia and then subsequently break up into smaller drops. In contrast, the smaller drops generated for slug flow conditions have lower initial radial velocities so that their lateral spread is limited to effects of turbulent dispersion based only on turbulence present in the gas phase.

\section{Liquid Volume Fractions and Fluxes}

Measured and predicted distributions of liquid volume fractions and fluxes for fully developed and slug flow are illustrated in Fig. 12. Measurements of liquid volume fractions include both present findings, using holography, and those of Ruff et al., ${ }^{1}$ using gamma-ray absorption. LHF predictions for slug flow are shown for reasonable limits of boundarylayer development along the injector passage, e.g., $L / d=0$ and 5 ; however, the differences between the two predictions are not very significant. The position of the liquid surface is also marked on the plots for reference purposes.

In Fig. 12, the liquid volume fraction measurements of Ruff et al. ${ }^{1}$ and the present investigation are only in fair agreement in the region where they overlap-particularly for fully developed flow. The problem is that both sets of measurements en- 


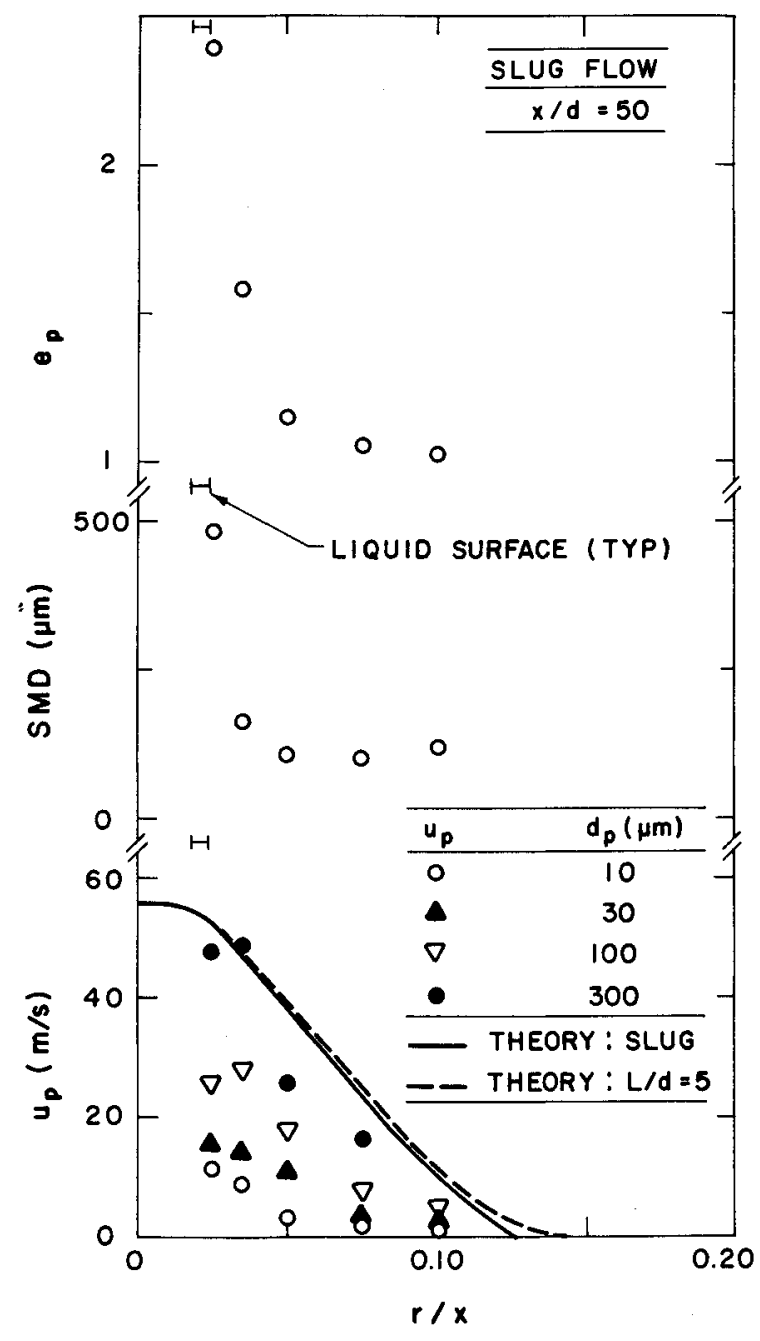

Fig. 10 Dispersed-phase properties for slug flow at $x / d=50$.

counter difficulties in this region: the gamma-ray absorption measurements of Ruff et al. ${ }^{1}$ are near the lower limit of resolution due to limitations of finite sampling times and signal-tonoise ratios, and the present measurements are also problematical due to difficulties of properly evaluating the volume of large and irregular liquid elements from their projected images-a problem that is more pronounced for fully developed flow where more irregular liquid elements are present. Even after accounting for this difficulty, it is evident that the multiphase mixing layer is quite dilute, quantifying the general impression obtained from the holograms, i.e., excluding the largest liquid elements, maximum liquid volume fractions are roughly 0.01 . For liquid volume fractions on this order, effects of collisions are not very significant. ${ }^{3}$

The results illustrated in Fig. 12 highlight the faster rate of radial mixing for fully developed flow than slug flow, e.g., fully developed flow exhibits higher liquid fluxes throughout the mixing layer, broader distributions of liquid volume fractions, and larger radial deflections of the liquid core. The main reason for this is the faster rate of breakup at the liquid surface for fully developed flow, promoted by disturbances due to turbulence in the liquid core. The ability of large liquid elements to penetrate across the mixing layer before subsequently breaking up also provides a mechanism for improved mixing, as noted earlier.

The LHF predictions of liquid volume fractions are reasonably good when liquid volume fractions are greater than 0.2 , as pointed out by Ruff et al. ${ }^{1}$ Present measurements in the mixing layer (where $\alpha_{f}$ is generally $<0.2$ ), however, are at best only qualitatively similar to the LHF predictions. In view of the velocity measurements illustrated in Figs. 4-11, which

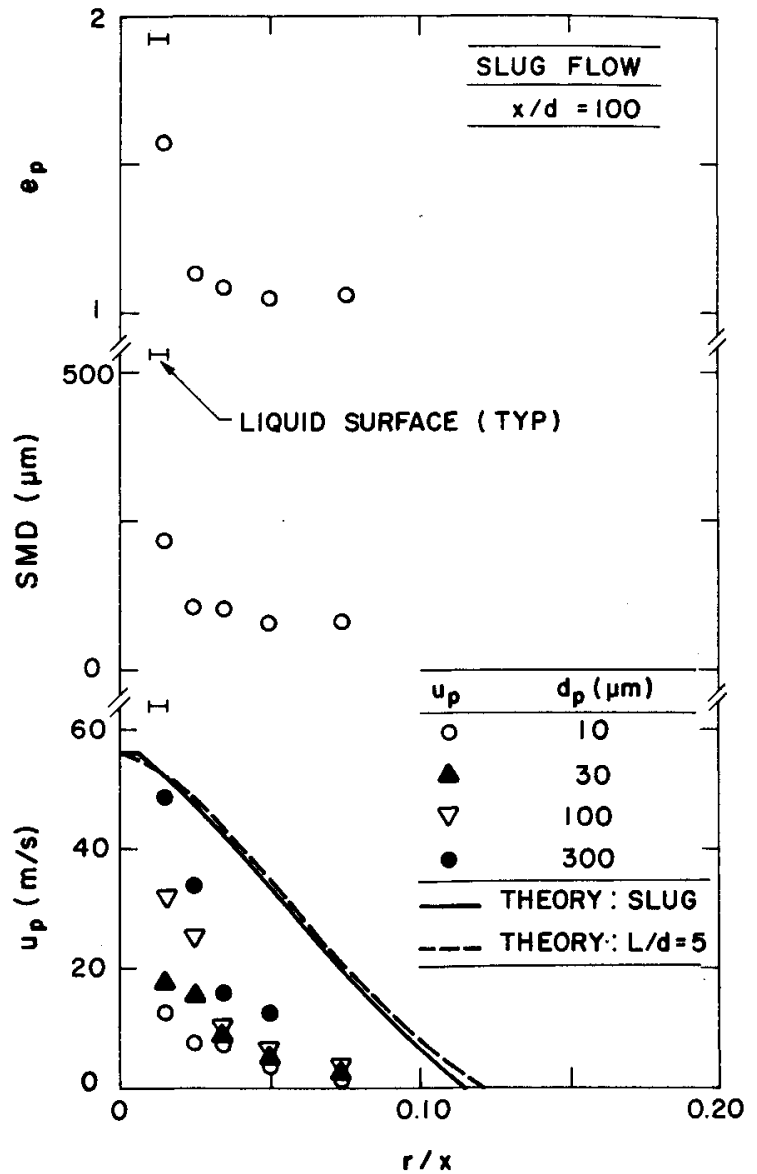

Fig. 11 Dispersed-phase properties for slug flow at $x / d=100$.

exhibit large differences in velocity for drops of different size, this is not surprising. The results also suggest that improved performance of the LHF approach for $\alpha_{f}>0.2$ does not result from the LHF approximation being formally satisfied. A more likely explanation is that the dynamics of the flow are dominated by the large liquid elements so that even though the gas and small drops are moving at much lower velocities, they do not have a significant effect. This follows since the mass fraction of gas and small drops is small due to the large density ratio of the flow (e.g., liquid volume fractions $>0.2$ generally correspond to mixture fractions $>0.99$ ) and the strong $d_{p}^{3}$ variation of drop mass with drop diameter.

\section{Discussion}

Measurements for present test conditions support the traditional view of spray structure, involving progressive breakup of larger to smaller liquid elements ${ }^{22}$; rather than recent hypotheses that small drops are either formed near the jet exit or are stripped from the surface of the liquid core and create larger drops within the multiphase mixing layer by collisions or coalescence. ${ }^{7,30,31}$ It appears that large liquid elements, like ligaments, are formed along the surface of the liquid core. These elements then break up into smaller liquid elements either due to the development of residual instabilities within the liquid elements that require additional time to complete their breakup action or by the development of subsequent instabilities as the liquid elements pass through the relatively slow-moving gas. Smaller drops resulting from breakup adjust more rapidly to local gas velocities so that the resulting reduced relative velocities, combined with larger relative stabilizing effects of surface tension, finally end the breakup process. The smaller drops then spread across the mixing layer through the mechanism of turbulent dispersion. Furthermore, it appears that final requirements of drop stability to subsequent breakup have an important effect on drop size distribu- 


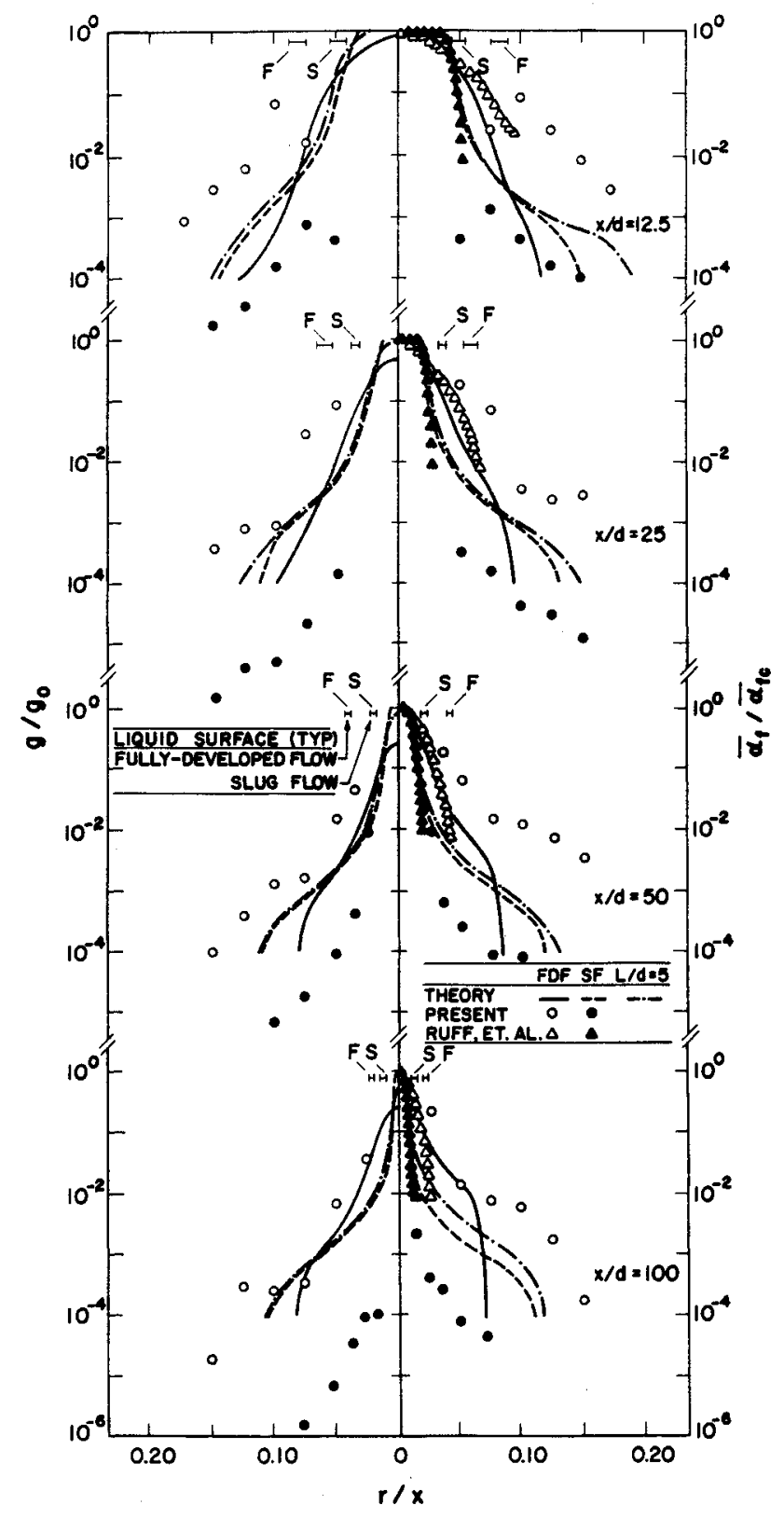

Fig. 12 Liquid volume fractions and fluxes at various distances from the injector for fully developed and slug flow.

tions produced in the present sprays. For example, drop sizes near the edge of the flow and at $x / d=100$ (see Figs. 4-11) are remarkably similar for slug and fully developed flow even though the nature of the dispersed phase near the liquid surface is very different.

Thus, the dense sprays observed for present test conditions do not correspond to closely spaced spherical elements dominated by collision processes; instead, they correspond to a relatively dilute flow of irregularly shaped liquid elements dominated by breakup processes. Current estimates of breakup times of drops suggest that they are comparable to mixing times in sprays, ${ }^{3}$ which is consistent with present observations. Thus, it is likely that analysis of breakup will have to be an integral part of analysis of dense sprays. Fortunately, the fact that the dense-spray region is optically accessible using high-resolution holography should be helpful for gaining a better understanding of the breakup processes relevant to this flow.

\section{Conclusions}

The near-injector region of pressure-atomized sprays was investigated, considering atomization breakup conditions with both slug and fully developed flow at the jet exit. The major conclusions of the study are as follows.

1) Drop velocities increase significantly with increasing drop size throughout the near-injector region, providing unequivocal evidence of the importance of separated-flow effects in dense sprays. Thus, the success of the LHF approximation at high liquid volume fractions is largely caused by the fact that the momentum of the gas and small drops does not have a strong influence on the dynamics of the flow since their momentum is a small fraction of the total momentum due to the large density ratio of the flow, even though they have significantly different velocities from the bulk of the liquid.

2) The multiphase mixing layer around the liquid core primarily consists of large irregularly shaped liquid elements and drops, near the core, with the proportion of drops increasing and drop sizes and mean liquid volume fractions decreasing with increasing radial distance. Even near the liquid core, however, the gas-containing region is relatively dilute at each instant. This structure suggests that breakup and turbulent dispersion of drops can be dominant processes in the multiphase mixing layer, rather than drop collisions as has been suggested in the past.

3) The degree of flow development and turbulence levels at the injector exit influences drop-size distributions near the liquid surface as well as mixing rates. Fully developed flow yields larger drops near the surface of the liquid core due to distortion and wrinkling of the surface by the liquid-phase turbulence, enhancing normal liquid-jet breakup mechanisms. Drop-size distributions near the edge of the flow and toward the downstream end of the dense-spray region, however, were not very different for slug or fully developed flow at the jet exit; this suggests that local stability of liquid elements to breakup has a strong influence on the eventual outcome of the atomization process.

4) High-resolution double-pulse holography is capable of penetrating the multiphase mixing layer to the surface of the liquid core as long as the object beam is not directed through the liquid core itself. This instrument provides a valuable approach for studying the properties of dense sprays since it can deal with irregularly shaped liquid elements that are problematical for other measurement techniques used in multiphase flows.

Present conclusions are based on measurements for large diameter $(9.5 \mathrm{~mm})$ water jets injected into still air at atmospheric pressure. Due to the sensitivity of dense sprays to jet exit and ambient conditions, generalization of these results to other conditions should be approached with caution. Additional measurements of dense-spray properties clearly are needed in order to gain a better understanding of this flow.

\section{Acknowledgments}

This research was sponsored by the Air Force Office of Scientific Research, Air Force Systems Command, U.S. Air Force, under Grant AFOSR-85-0244. The image analysis system used during the investigation was purchased under Grant MSM-850634 from the National Science Foundation.

\section{References}

${ }^{1}$ Ruff, G. A., Sagar, A. D., and Faeth, G. M., "Structure and Mixing Properties of Pressure-Atomized Sprays,"' AIAA Journal, Vol. 27, No. 7, 1989, pp. 901-909.

${ }^{2}$ Ranz, W. E., "Some Experiments on Orifice Sprays," Canadian Journal of Chemical Engineering, Vol. 36, Aug. 1958, pp. 175-181.

${ }^{3}$ Faeth, G. M., "Mixing, Transport and Combustion in Sprays," Progress in Energy Combustion Science, Vol. 13, No. 3, 1987, pp. 293-345.

${ }^{4}$ Phinney, R. E., "The Breakup of a Turbulent Liquid Jet in a Gaseous Atmosphere," Journal of Fluid Mechanics, Vol. 60, Part 4, Oct. 1973, pp. 689-701. 
${ }^{5}$ Hiroyasu, H., Shimizu, M., and Arai, M., "The Breakup of a High Speed Jet in a High Pressure Gaseous Environment," ICLASS82, Univ. of Wisconsin, Madison, WI, 1982.

${ }^{6}$ Chehroudi, B., Onuma, Y., Chen, S.-H., and Bracco, F. V., "On the Intact Core of Full-Cone Sprays," Society of Automotive Engineers, Warrendale, PA, Paper 850126, 1985.

"Bracco, F. V., Structure of High-Speed Full-Cone Sprays," Recent Advances in Gas Dynamics, edited by C. Casci, Plenum, New York, 1983.

${ }^{8}$ Wu, K.-J., Su, C.-C., Steinberger, R. L., Santavicca, D. A., and Bracco, F. V., "Measurements of the Spray Angle of Atomizing Jets," Journal of Fluids Engineering, Vol. 105, No. 4, Dec. 1983, pp. 406-415.

${ }^{9}$ Wu, K.-J., Coghe, A., Santavicca, D. A., and Bracco, F. V., "LDV Measurements of Drop Velocity in Diesel-Type Sprays," AIAA Journal, Vol. 22, No. 9, 1984, pp. 1263-1270.

${ }^{10}$ Mao, C.-P., Wakamatsu, Y., and Faeth, G. M., "A Simplified Model of High Pressure Spray Combustion," Eighteenth Symposium (International) on Combustion, Combustion Institute, Pittsburgh, PA, 1980, pp. 337-347.

${ }^{11}$ Mao, C.-P., Szekely, G. A., Jr., and Faeth, G. M., "Evaluation of a Locally Homogeneous Flow Model of Spray Combustion," Journal of Energy, Vol. 4, March-April 1980, No. 2, pp. 78-87.

${ }^{12}$ Shearer, A. J., Tamura, H., and Faeth, G. M., "Evaluation of a Locally Homogeneous Flow Model of Spray Evaporation, Journal of Energy, Vol. 3, Sept.-Oct. 1979, No. 5, pp. 271-278.

${ }^{13}$ Solomon, A. S. P., Shuen, J.-S., Zhang, Q.-F., and Faeth, G. M., "Structure of Nonevaporating Sprays: I. Near-Injector Conditions and Mean Properties," AIAA Journal, Vol. 23, No. 10, 1985, pp. 1548-1555.

${ }^{14}$ Solomon, A. S. P., Shuen, J.-S., Zhang, Q.-F., and Faeth, G. M., "Structure of Nonevaporating Sprays: II. Drop and Turbulence Properties," AIAA Journal, Vol. 23, No. 11, 1985, pp. 1724-1730.

${ }^{15}$ Shuen, J.-S., Chen, L.-D., and Faeth, G. M., "Evaluation of a Stochastic Model of Particle Dispersion in a Turbulent Round Jet," AIChE Journal, Vol. 29, Jan. 1983, No. 1, pp. 167-170.

${ }^{16}$ Shuen, J.-S., Chen, L.-D., and Faeth, G. M., "Predictions of the Structure of Turbulent, Particle-Laden, Round Jets," AIAA Journal, Vol. 21, No. 11, 1983, pp. 1483-1484.

${ }^{17}$ Shuen, J.-S., Solomon, A. S. P., Zhang, Q.-F., and Faeth, G.
M., "Structure of Particle-Laden Jets: Measurements and Predictions," AIAA Journal, Vol. 23, No. 3, 1985, pp. 396-404.

${ }^{18}$ Reitz, R. D., and Bracco, F. V., "Mechanism of Atomization of a Liquid Jet," Physics of Fluids, Vol. 25, No. 10, Oct. 1982, pp. 1730-1742.

${ }^{19}$ Reitz, R. D., "Atomization and Other Breakup Regimes of a Liquid Jet," Ph.D. Dissertation, Princeton Univ., Princeton, NJ, 1978.

${ }^{20}$ Ruff, G. A., and Faeth, G. M., "Dense-Spray Structure and Phenomena: Part II. Pressure-Atomized Sprays," Grant AFOSR-850244, University of Michigan, Ann Arbor, MI, Interim Rept., Sept. 1987.

${ }^{21}$ Ruff, G. A., "Structure and Mixing Properties of the NearInjector Region of Nonevaporating Pressure-Atomized Sprays," Ph.D. Dissertation, Univ. of Michigan, Ann Arbor, MI, 1990.

${ }^{22}$ Lefebvre, A. H., "Atomization," Progress in Energy Combustion Science, Vol. 6, No. 3, 1980, pp. 223-246.

${ }^{23}$ Hinze, J. O., Turbulence, 2nd ed., McGraw-Hill, New York, 1975 , pp. 427, 724-734.

${ }^{24}$ Schlichting, H., Boundary Layer Theory, 7th ed., McGraw-Hill, New York, 1979, p. 599.

${ }^{25}$ Miesse, C. C., "Correlation of Experimental Data on Disintegration of Liquid Jets,"' Industrial and Engineering Chemistry, Vol. 47, No. 3, Sept. 1955, pp. 1690-1697.

${ }^{26}$ Lockwood, F. C., and Naguib, A. S., "The Prediction of Fluctuations in the Properties of Free, Round-Jet Turbulent Diffusion Flames," Combustion and Flame, Vol. 24, No. 1, Feb. 1975, pp. 109-124.

${ }^{27}$ Bilger, R. W., "Turbulent Jet Diffusion Flames," Progress in Energy Combustion Science, Vol. 1, No. 1, 1976, pp. 87-109.

${ }^{28}$ Jeng, S.-M., and Faeth, G. M., "Species Concentrations and Turbulence Properties in Buoyant Methane Diffusion Flames," Journal of Heat Transfer, Vol. 106, No. 3, Aug. 1985, pp. 721-727.

${ }^{29}$ Spalding, D. B., GENMIX: A General Computer Program for Two-Dimensional Parabolic Phenomena, Pergamon, Oxford, 1977.

${ }^{30}$ Reitz, R. D., and Bracco, F. V., "Mechanisms of Breakup of Round Liquid Jets," Encyclopedia of Fluid Mechanics, Vol. III, edited by N. P. Cheremisinoff, 1984, Chap. 11.

${ }^{31}$ Reitz, R. D., and Diwakar, R., "'Structure of High-Pressure Fuel Sprays," Society of Automotive Engineers, Warrendale, PA, Paper $870598,1987$. 